INTERNATIONAL RESEARCH JOURNAL OF PHARMACY

www.irjponline.com

ISSN $2230-8407$

\title{
Research Article \\ DETERMINATION OF BIOACTIVE COMPONENTS OF MYXOPYRUM SERRATULUM A.W. HILL (OLEACEAE) STEM BY GC-MS ANALYSIS
}

K. Rajalakshmi, V. R. Mohan*

Ethnopharmacology Unit. Research Department of Botany, V.O.Chidambaram College, Tuticorin, Tamil Nadu. India

*Corresponding Author Email: vrmohanvoc@gmail.com

Article Received on: 21/04/16 Revised on: 04/05/16 Approved for publication: 17/05/16

DOI: $10.7897 / 2230-8407.07782$

\begin{abstract}
The present study is to evaluate bioactive compounds of ethanol extract of Myxopyrum serratulum stem using GC-MS analysis. The chemical compositions of the ethanol extract of stem of Myxopyrum serratulum were investigated using Perkin-Elmer Gas Chromatography-Mass Spectrometry, while the mass spectra of the compounds found in the extract were matched with the National Institute of Standard and Technology (NIST) library. Thirty two compounds were identified. The prevailing compounds in ethanol extract were 2-Propenoic acid, 3-(4-methoxyphenyl)-, $\alpha$ Amyrin, 1-(2-Hydroxy-ethyl)-2-methyl-1H-benzoimidazole-5-carboxylic acid methyl ester, n-Hexadecanoic acid, Pyrazolidine-3,5-dione and 4phenyl-, 5-Hydroxymethylfurfural. The compounds identified through the GC-MS analysis were used in various applications as antimicrobial, antiinflammatory, hepatoprotective and cancer preventive.
\end{abstract}

Keywords: Myxopyrum serratulum, GC-MS, Phytocomponent, Phytol, 5-Hydroxymethylfurfural, 2-Propenoic acid, 3-(4-methoxyphenyl)-, $\alpha$ Amyrin.

\section{INTRODUCTION}

There is an increasing interest in the phytochemical compounds, which could be relevant to their nutritional incidence and their role in health and disease ${ }^{1}$. In the recent years, the interest for the study of the organic compounds from plants and their activity has increased. A lot of extraction methods and analytical methods like spectrophotometry, high performance liquid chromatography, capillary electrophoresis (HPLC), gas chromatography-flame ionization detection (GC-FID), gas chromatography-mass spectrometry (GC-MS) are developed for the study about plant active compounds ${ }^{2,3}$. The aim of the present study is to develop a rapid method for quantitative determination of organic compounds in herbs by GC-MS technique.

Myxopyrum serratulum (Oleaceae) commonly known as "Chaturamulla" is a large woody climbing shrub. The leaves are used as astringent, acrid, sweet, thermogenic, anodyne, febrifuge and tonic. They are useful in vitiated conditions of kapha and vata, cough, asthma, rheumatism, cephalalgia, nostalagia, consumption, fever, otopathy, neuropathy and cuts and wounds ${ }^{4}$. Taking into consideration of the medicinal importance of this plant, the ethanol extract of stem of M. serratulum was analysed for the first time using GC-MS. This work will help to identify the compounds of therapeutic value. A majority of the rich diversity of southern region of Western Ghats Tamil Nadu medicinal plants are yet to be scientifically evaluated for such properties. With this background, the present study was aimed to identify the phytoconstituents present in $M$. serratulum using GC-MS analysis. GC-MS is the best technique to identify the bioactive constituents of long chain hydrocarbons, alcohols, acids esters, alkaloids, steroids, amino acid and nitro compounds ${ }^{5}$.

\section{MATERIALS AND METHODS}

\section{Collection of plant sample}

The stem of Myxopyrum serratulum A.W. Hill were collected from Pechiparai, Kanyakumari District, Tamil Nadu. With the help of local flora, the specimens were identified and preserved in the Ethnopharmacology Unit, Research Department of Botany, V.O. Chidambaram College, Tuticorin, Tamil Nadu.

\section{Preparation of plant extract}

The stem of Myxopyrum serratulum were cleaned, shade dried and pulverized to powder in a mechanical grinder. Required quantity of powder was weighed and transferred to stoppered flask, and treated with ethanol until the powder is fully immersed. The flask was shaken every hour for the firsts 6 hours and then it was kept aside and again shaken after 24 hours. This process was repeated for 3 days and then the extract was filtered. The extract was collected and evaporated to dryness by using vacuum distillation unit. The final residue thus obtained was then subjected to GC-MS analysis.

\section{GC-MS Analysis}

GC-MS analysis of ethanol extract was performed with GC clarus 500 Perkin Elmer system and Gas chromatograph interfaced to a Mass spectrometer (GC-MS) equipped with a Elite -1 fused silica capillary column $(30 \mathrm{~mm} \times 0.25 \mathrm{~mm} 1 \mathrm{D} \mathrm{x}$ $1 \mathrm{um}$ df, composed of $100 \%$ Dimethyl poly siloxane). For GCMS detection, and electron ionization system with ionizing energy of $70 \mathrm{eV}$ was used. Helium gas $(99.999 \%)$ was used as the carrier gas at constant flow rate $1 \mathrm{ml} / \mathrm{min}$ and an injection volume of 2 ul was employed (Split ratio of 10:1); Injector temperature $250^{\circ} \mathrm{C}$; ion-source temperature $280^{\circ} \mathrm{C}$. The oven 
temperature was programmed from $110^{\circ} \mathrm{C}$ (isothermal for 2 min) with an increase of $10^{\circ} \mathrm{C} / \mathrm{min}$, to $200^{\circ} \mathrm{C}$, then $5^{\circ} \mathrm{C} / \mathrm{min}$ to $280^{\circ} \mathrm{C}$, ending with a $9 \mathrm{~min}$ isothermal at $280^{\circ} \mathrm{C}$. Mass spectra were taken at $70 \mathrm{eV}$, a scan interval of 0.5 seconds and fragments from 45 to $450 \mathrm{Da}$. Total $\mathrm{GC}$ running time was 36 minutes. The relative \% amount of each component was calculated by comparing its average peak area to the total areas, software adopted to handle mass spectra and chromatograms was a turbomass.

Interpretation on mass spectrum of GC-MS was done using the database of National Institute of Standard and Technology (NIST) having more than 62,000 patterns. The mass spectrum of the unknown component was compared with the spectrum of the known components stored in the NIST library. The name, molecular weight and structure of the components of the test materials were ascertained.

\section{RESULTS}

The results pertaining to GC-MS analysis led to the identification of number of compounds from the GC fraction of the ethanol extract of Myxopyrum serratulum stem. These compounds were identified through mass spectrometry attached with GC. The compounds present in the ethanol extract of $M$. serratulum stem identified by GC-MS analysis are shown in Figure 1. The active principles with their retention time (RT), molecular formula, molecular weight, (MW), and concentration $(\%)$ in the ethanol extract of $M$. serratulum stem are presented in table 1 . The prevailing compounds in ethanol extract were 2Propenoic acid, 3-(4-methoxyphenyl)- (25.87), $\alpha$-Amyrin (13.71), 1-(2-Hydroxy-ethyl)-2-methyl-1H-benzoimidazole-5carboxylic acid methyl ester (10.96), n-Hexadecanoic acid (7.63), Pyrazolidine-3,5-dione, 4-phenyl- (5.82), 5Hydroxymethylfurfural (4.66), (3-Nitrophenyl) methanol, isopropyl ether (3.68), 9-Octadecenoic acid, (E)- (3.35), 9,12Octadecadienoic acid (Z,Z)- (3.16), ß-Amyrin (2.68), Phytol (2.59), Isoquinoline, 1,2,3,4-tetrahydro-1-allyl-6,7-dimethoxy3,3-dimethyl- (2.13), 5-Hydroxy-6-methyl-12,13-dioxatricyclo[7.3.1.0(1,6)]tridecane-8-carboxylic acid, methyl ester (1.80), Ethyl $\alpha$-d-glucopyranoside (1.79), 4-((1E)-3-Hydroxy-1propenyl)-2-methoxyphenol (1.41) respectively. Figure $2,3,4,5,6,7,8,9,10,11,12,13$ and 14 show the mass spectrum and structures of D-Mannopyranose, Ethyl hydrogen succinate, (3Nitrophenyl) methanol, isopropyl ether, Pyrazolidine-3,5-dione, 4-phenyl-, 1-(2-Hydroxy-ethyl)-2-methyl-1H-benzoimidazole-5carboxylic acid methyl ester, Galactitol, Phytol, 9-Octadecenoic acid, (E)-, Oleic Acid, Betulin, $\beta$-Amyrin, $\alpha$-Amyrin, 9,19Cycloergost-24(28)-en-3-ol, 4,14-dimethyl-, acetate, $(3 \beta, 4 \alpha, 5 \alpha)$. Table 2 listed the major phytocomponents and their biological activities obtained through the GC-MS study of $M$. serratulum stem.

\begin{tabular}{|c|c|c|c|c|}
\hline RT & Name of the compound & $\begin{array}{l}\text { Molecular } \\
\text { Formula }\end{array}$ & $\begin{array}{l}\text { Molecular } \\
\text { Weight }\end{array}$ & $\begin{array}{c}\text { Peak } \\
\text { Area \% }\end{array}$ \\
\hline 4.47 & D-Mannopyranose & $\mathrm{C}_{6} \mathrm{H}_{12} \mathrm{O}_{6}$ & 180 & 0.63 \\
\hline 4.72 & Ethyl hydrogen succinate & $\mathrm{C}_{6} \mathrm{H}_{10} \mathrm{O}_{4}$ & 146 & 0.75 \\
\hline 5.83 & 5-Hydroxymethylfurfural & $\mathrm{C}_{6} \mathrm{H}_{6} \mathrm{O}_{3}$ & 126 & 4.66 \\
\hline 6.06 & 7-Ethyl-4-decen-6-one & $\mathrm{C}_{12} \mathrm{H}_{22} \mathrm{O}$ & 182 & 0.17 \\
\hline 6.48 & 3-Hydroxydecanoic acid & $\mathrm{C}_{10} \mathrm{H}_{20} \mathrm{O}_{3}$ & 188 & 0.52 \\
\hline 9.14 & (3-Nitrophenyl) methanol, isopropyl ether & $\mathrm{C}_{10} \mathrm{H}_{13} \mathrm{NO}_{3}$ & 195 & 3.68 \\
\hline 9.77 & Pyrazolidine-3,5-dione, 4-phenyl- & $\mathrm{C}_{9} \mathrm{H}_{8} \mathrm{~N}_{2} \mathrm{O}_{2}$ & 176 & 5.82 \\
\hline 10.59 & Methyl trans-4-methylcinnamate & $\mathrm{C}_{11} \mathrm{H}_{12} \mathrm{O}_{2}$ & 176 & 0.60 \\
\hline 11.63 & Ethyl $\alpha$-d-glucopyranoside & $\mathrm{C}_{8} \mathrm{H}_{16} \mathrm{O}_{6}$ & 208 & 1.79 \\
\hline 12.53 & 2-Propenoic acid, 3-(4-methoxyphenyl)- & $\mathrm{C}_{10} \mathrm{H}_{10} \mathrm{O}_{3}$ & 178 & 25.87 \\
\hline 12.83 & 4-((1E)-3-Hydroxy-1-propenyl)-2-methoxyphenol & $\mathrm{C}_{10} \mathrm{H}_{12} \mathrm{O}_{3}$ & 180 & 1.41 \\
\hline 14.00 & 3,7,11,15-Tetramethyl-2-hexadecen-1-ol & $\mathrm{C}_{20} \mathrm{H}_{40} \mathrm{O}$ & 296 & 0.87 \\
\hline 15.16 & 1-(2-Hydroxy-ethyl)-2-methyl-1H-benzoimidazole-5-carboxylic acid methyl ester & $\mathrm{C}_{12} \mathrm{H}_{14} \mathrm{~N}_{2} \mathrm{O}_{3}$ & 234 & 10.96 \\
\hline 15.62 & n-Hexadecanoic acid & $\mathrm{C}_{16} \mathrm{H}_{32} \mathrm{O}_{2}$ & 256 & 7.63 \\
\hline 15.76 & Isoquinoline, 1,2,3,4-tetrahydro-1-allyl-6,7-dimethoxy-3,3-dimethyl- & $\mathrm{C}_{16} \mathrm{H}_{23} \mathrm{NO}_{2}$ & 261 & 2.13 \\
\hline 16.47 & Galactitol & $\mathrm{C}_{6} \mathrm{H}_{14} \mathrm{O}_{6}$ & 182 & 0.22 \\
\hline 17.69 & Phytol & $\mathrm{C}_{20} \mathrm{H}_{40} \mathrm{O}$ & 296 & 2.59 \\
\hline 18.01 & 9,12-Octadecadienoic acid (Z,Z)- & $\mathrm{C}_{18} \mathrm{H}_{32} \mathrm{O}_{2}$ & 280 & 3.16 \\
\hline 18.11 & 9-Octadecenoic acid, (E)- & $\mathrm{C}_{18} \mathrm{H}_{34} \mathrm{O}_{2}$ & 282 & 3.35 \\
\hline 18.44 & Octadecanoic acid & $\mathrm{C}_{18} \mathrm{H}_{36} \mathrm{O}_{2}$ & 284 & 1.02 \\
\hline 18.50 & Oleic Acid & $\mathrm{C}_{18} \mathrm{H}_{34} \mathrm{O}_{2}$ & 282 & 0.32 \\
\hline 19.55 & $\begin{array}{l}\text { 5-Hydroxy-6-methyl-12,13-dioxa-tricyclo[7.3.1.0(1,6)]tridecane-8-carboxylic acid, } \\
\text { methyl ester }\end{array}$ & $\mathrm{C}_{14} \mathrm{H}_{22} \mathrm{O}_{5}$ & 270 & 1.80 \\
\hline 20.74 & E)-13-Docosenoic acid & $\mathrm{C}_{22} \mathrm{H}_{42} \mathrm{O}_{2}$ & 338 & 0.36 \\
\hline 24.53 & Betulin & $\mathrm{C}_{30} \mathrm{H}_{50} \mathrm{O}_{2}$ & 442 & 0.62 \\
\hline 26.16 & 9-Octadecenoic acid (Z)-, 2-hydroxy-1-(hydroxymethyl)ethyl ester & $\mathrm{C}_{21} \mathrm{H}_{40} \mathrm{O}_{4}$ & 356 & 0.11 \\
\hline 27.22 & $\beta$-Amyrin & $\mathrm{C}_{30} \mathrm{H}_{50} \mathrm{O}$ & 426 & 2.68 \\
\hline 29.15 & $\alpha$-Amyrin & $\mathrm{C}_{30} \mathrm{H}_{50} \mathrm{O}$ & 426 & 13.71 \\
\hline 30.54 & Quinoline, 3-dodecyl-2-methyl-4-[(4-methoxyphenyl)methoxy]- & $\mathrm{C}_{30} \mathrm{H}_{41} \mathrm{NO}_{2}$ & 447 & 1.00 \\
\hline 32.96 & dl- $\alpha$-Tocopherol & $\mathrm{C}_{29} \mathrm{H}_{50} \mathrm{O}_{2}$ & 430 & 0.24 \\
\hline 34.37 & $\begin{array}{l}\text { 1b,4a-Epoxy-2H-cyclopenta[3,4]cyclopropa[8,9]cycloundec[1,2-b]oxiren-5(6H)-one, } \\
\text { 7-(acetyloxy)decahydro-2,9,10-trihydroxy-3,6,8,8,10a-pentamethyl- }\end{array}$ & $\mathrm{C}_{22} \mathrm{H}_{32} \mathrm{O}_{8}$ & 424 & 0.20 \\
\hline 34.86 & 6,7-Epoxypregn-4-ene-9,11,18-triol-3,20-dione, 11,18-diacetate & $\mathrm{C}_{25} \mathrm{H}_{32} \mathrm{O}_{8}$ & 460 & 0.70 \\
\hline 35.75 & 9,19-Cycloergost-24(28)-en-3-ol, 4,14-dimethyl-, acetate, $(3 \beta, 4 \alpha, 5 \alpha)$ - & $\mathrm{C}_{32} \mathrm{H}_{52} \mathrm{O}_{2}$ & 468 & 0.39 \\
\hline
\end{tabular}


Table 2: Activity of phytocomponents identified in the ethanol extract of $M$. serratulum stem

\begin{tabular}{|c|c|c|c|}
\hline No. & Name of the compound & Compound Nature & **Activity \\
\hline 1. & D-Mannopyranose & Sugar moiety & Preservative \\
\hline 2. & Ethyl hydrogn succinate & $\begin{array}{l}\text { Succinic acid } \\
\text { compound }\end{array}$ & Antimicrobial \\
\hline 3. & 5-Hydroxymethylfurfural & Aldehyde compound & Antimicrobial, Antiinflammatory \\
\hline 4. & 7-Ethyl-4-decen-6-one & Ketone compound & No activity is reported \\
\hline 5. & 3-Hydroxydecanoic acid & $\begin{array}{l}\text { Alcoholic } \\
\text { compound }\end{array}$ & Antimicrobial \\
\hline 6. & (3-Nitrophenyl) methanol, isopropyl ether & Nitrogen compound & Antimicrobial \\
\hline 7. & Pyrazolidine-3,5-dione, 4-phenyl- & Alkaloid & Antimicrobial, Anti-inflammatory \\
\hline 8. & Methyl trans-4-methylcinnamate & $\begin{array}{l}\text { Cinnamic acid } \\
\text { compound }\end{array}$ & Antimicrobial, Anti-inflammatory \\
\hline 9. & Ethyl $\alpha$-d-glucopyranoside & Sugar moiety & Preservative \\
\hline 10. & 2-Propenoic acid, 3-(4-methoxyphenyl)- & Aromatic compound & No activity reported \\
\hline 11. & 4-((1E)-3-Hydroxy-1-propenyl)-2-methoxyphenol & $\begin{array}{l}\text { Phenolic } \\
\text { compound }\end{array}$ & $\begin{array}{l}\text { Antimicrobial, Anti-inflammatory } \\
\text { Antioxidant, Analgesic }\end{array}$ \\
\hline 12. & 3,7,11,15-Tetramethyl-2-hexadecen-1-ol & Terpene alcohol & Antimicrobial, Anti-inflammatory \\
\hline 13. & $\begin{array}{l}\text { 1-(2-Hydroxy-ethyl)-2-methyl-1H-benzoimidazole-5- } \\
\text { carboxylic acid methyl ester }\end{array}$ & Amino compound & Antimicrobial, Anti-inflammatory \\
\hline 14. & n-Hexadecanoic acid & Palmitic acid & $\begin{array}{l}\text { Antioxidant, Hypocholesterolemic } \\
\text { Nematicide, Pesticide, Lubricant } \\
\text { Antiandrogenic, Flavor, Hemolytic }\end{array}$ \\
\hline 15. & $\begin{array}{l}\text { Isoquinoline, 1,2,3,4-tetrahydro-1-allyl-6,7-dimethoxy-3,3- } \\
\text { dimethyl- }\end{array}$ & Alkaloid & Antimicrobial, Anti-inflammatory \\
\hline 16. & Galactitol & Sugar alcohol & Leads to cataract \\
\hline 17. & Phytol & Diterpene & $\begin{array}{l}\text { Antimicrobial, Anti-inflammatory } \\
\text { Anticancer, Diuretic }\end{array}$ \\
\hline 18. & 9,12-Octadecadienoic acid (Z,Z)- & Linoleic acid & $\begin{array}{l}\text { Antiinflammatory, Hypocholesterolemic } \\
\text { Cancer preventive, Hepatoprotective, } \\
\text { Nematicide, Insectifuge, Antihistaminic, } \\
\text { Antieczemic, Antiacne, 5-Alpha reductase } \\
\text { inhibitor Antiandrogenic, Antiarthritic, } \\
\text { Anticoronary, Insectifuge }\end{array}$ \\
\hline 19. & 9-Octadecenoic acid, (E)- & $\begin{array}{l}\text { Oleic acid } \\
\text { compound }\end{array}$ & $\begin{array}{l}\text { Cancer preventive, Flavor } \\
\text { Hypocholesterolemic, 5-Alpha reductase } \\
\text { inhibitor, Antiandrogenic, Perfumery } \\
\text { Insectifuge, Anti-inflammatory } \\
\text { Anemiagenic, Dermatitigenic, Choleretic }\end{array}$ \\
\hline 20. & Octadecanoic acid & Stearic acid & No activity reported \\
\hline 21. & Oleic Acid & $\begin{array}{l}\text { Oleic acid } \\
\text { compound }\end{array}$ & $\begin{array}{l}\text { Cancer preventive, Flavor, } \\
\text { Hypocholesterolemic } \\
\text { 5-Alpha reductase inhibitor, Antiandrogenic } \\
\text { Perfumery, Insectifuge, Anti-inflammatory, } \\
\text { Anemiagenic, Dermatitigenic, Choleretic }\end{array}$ \\
\hline 22. & $\begin{array}{l}\text { 5-Hydroxy-6-methyl-12,13-dioxa- } \\
\text { tricyclo[7.3.1.0(1,6)]tridecane-8-carboxylic acid, methyl ester }\end{array}$ & Ester compound & No activity reported \\
\hline 23. & E)-13-Docosenoic acid & $\begin{array}{l}\text { Unsaturated fatty } \\
\text { acid }\end{array}$ & No activity reported \\
\hline 24. & Betulin & Triterpene & $\begin{array}{l}\text { Antibacterial, Antioxidant, Antitumor } \\
\text { Cancer preventive, Antiviral, Antiflu } \\
\text { Cytotoxic, Anti HIV, Anticarcinomic } \\
\text { Anti-inflammatory, Antifeedant } \\
\end{array}$ \\
\hline 25. & $\begin{array}{l}\text { 9-Octadecenoic acid (Z)-, 2-hydroxy-1-(hydroxymethyl)ethyl } \\
\text { ester }\end{array}$ & $\begin{array}{l}\text { Oleic acid } \\
\text { compound }\end{array}$ & No activity reported \\
\hline 26. & $\beta$-Amyrin & Triterpene & $\begin{array}{l}\text { Antibacterial, Antioxidant } \\
\text { Antitumor, Cancer preventive } \\
\text { Immunostimulant, Chemo preventive } \\
\text { Lipoxygenase-inhibitor, Pesticide }\end{array}$ \\
\hline 27. & $\alpha$-Amyrin & Triterpene & $\begin{array}{l}\text { Antibacterial, Antioxidant } \\
\text { Antitumor, Cancer preventive } \\
\text { Immunostimulant, Chemo preventive } \\
\text { Lipoxygenase-inhibitor, Pesticide }\end{array}$ \\
\hline 28. & $\begin{array}{l}\text { Quinoline, 3-dodecyl-2-methyl-4-[(4- } \\
\text { methoxyphenyl)methoxy]- }\end{array}$ & Alkaloid & Antimicrobial, Anti-inflammatory \\
\hline 29. & dl- $\alpha$-Tocopherol & $\begin{array}{l}\text { Vitamin E } \\
\text { compound }\end{array}$ & $\begin{array}{l}\text { Antiageing, Analgesic, Antidiabatic, } \\
\text { Antiinflammatory, Antioxidant, } \\
\text { Antidermatitic, Antileukemic, Antitumor, } \\
\text { Anticancer, Hepatoprotective, } \\
\text { Hypocholesterolemic, Antiulcerogenic, } \\
\text { Vasodilator, Antispasmodic, Antibronchitic, } \\
\text { Anticoronary }\end{array}$ \\
\hline
\end{tabular}




\begin{tabular}{|c|l|l|l|}
\hline \hline 30. & $\begin{array}{l}1 \text { b,4a-Epoxy-2H- } \\
\text { cyclopenta[3,4]cyclopropa[8,9]cycloundec[1,2-b]oxiren- } \\
5(6 \mathrm{H}) \text {-one, 7-(acetyloxy)decahydro-2,9,10-trihydroxy- } \\
\\
3,6,8,8,10 \mathrm{a}-\text {-pentamethyl- }\end{array}$ & Oxiren compound & No activity reported \\
\hline 31. & $\begin{array}{l}6,7-\text {-Epoxypregn-4-ene-9,11,18-triol-3,20-dione, 11,18- } \\
\text { diacetate }\end{array}$ & Ketone compound & No activity reported \\
\hline 32. & $\begin{array}{l}9,19-\text {-Cycloergost-24(28)-en-3-ol, 4,14-dimethyl-, acetate, } \\
(3 \beta, 4 \alpha, 5 \alpha)-\end{array}$ & Steroid & $\begin{array}{l}\text { Antimicrobial, Anti-inflammatory } \\
\text { Anticancer, Antiasthma } \\
\text { Hepatoprotective, Diuretic }\end{array}$ \\
\hline
\end{tabular}

**Source: Dr.Duke's Phytochemical and Ethnobotanical Databases

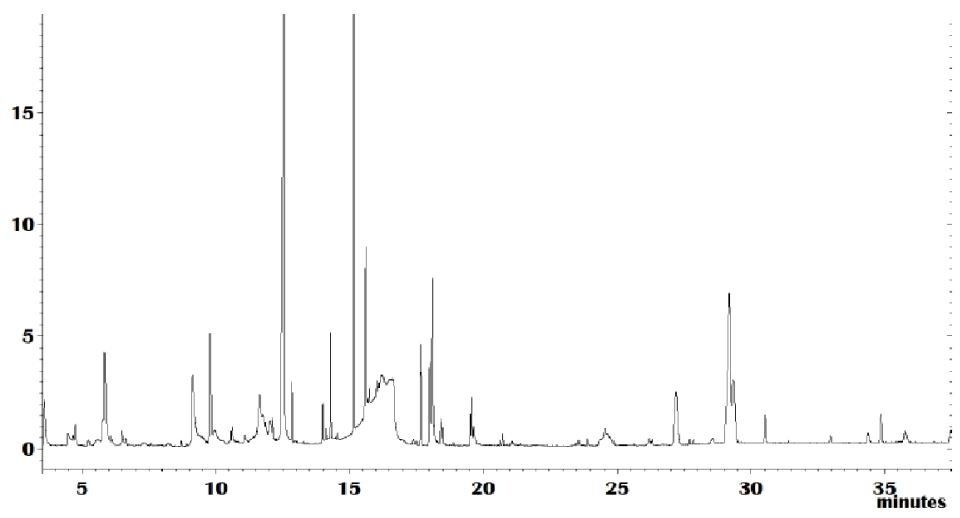

Figure 1: GC-MS chromatogram of the ethanol extract of $M$. serratulum stem

Mass Spectrum of Myxopyrum serratulum stem

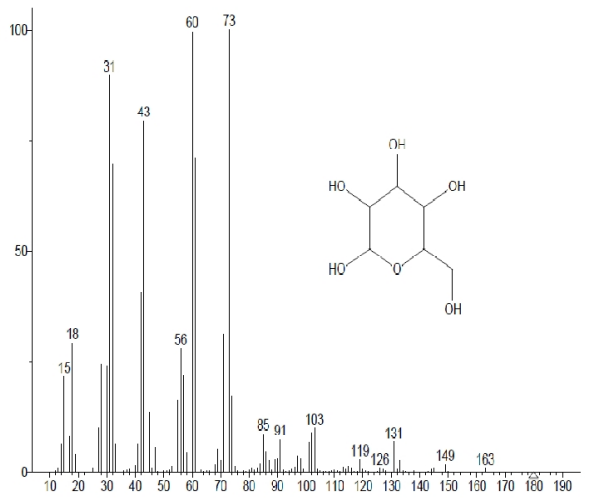

Figure 2: D-Mannopyranose

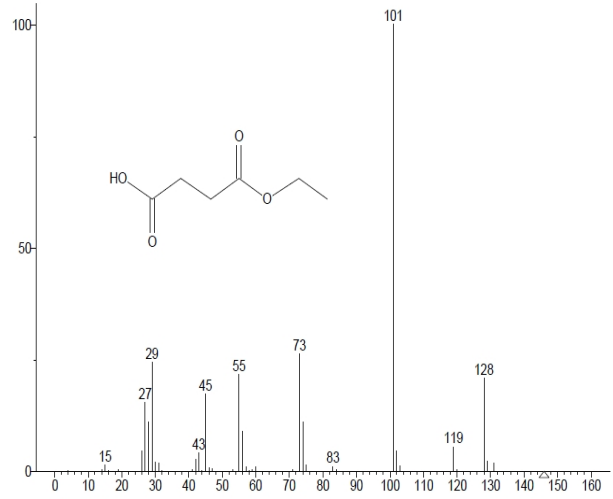

Figure 3: Ethyl hydrogn succinate

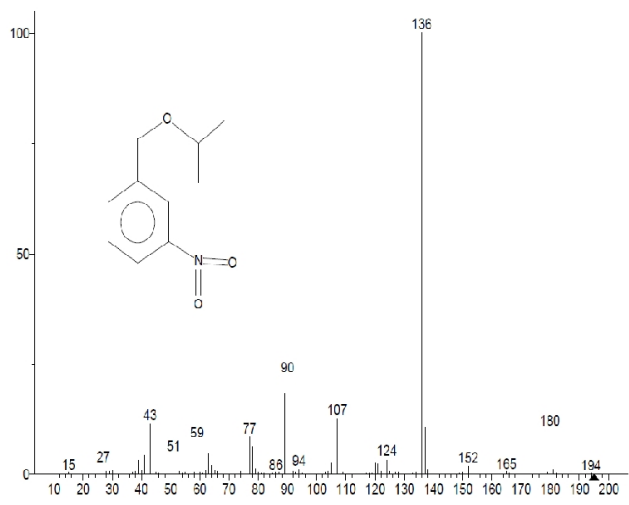

Figure 4: (3-Nitrophenyl) methanol, isopropyl ether 


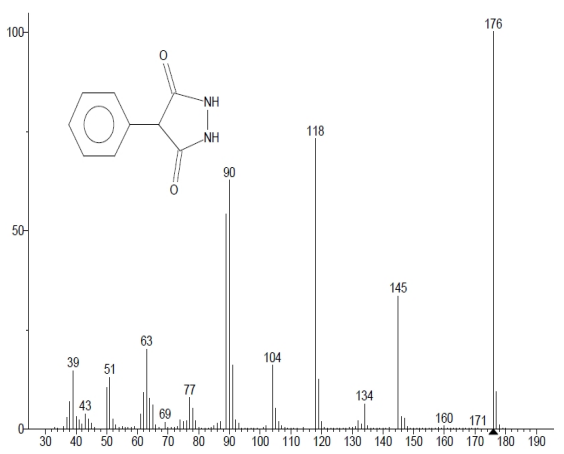

Figure 5: Pyrazolidine-3,5-dione, 4- phenyl

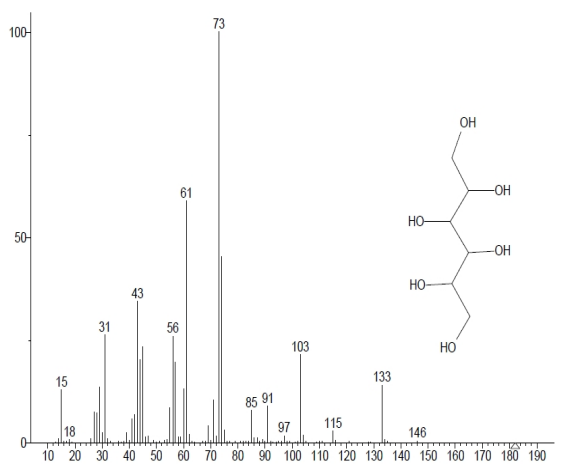

Figure 7: Galactitol

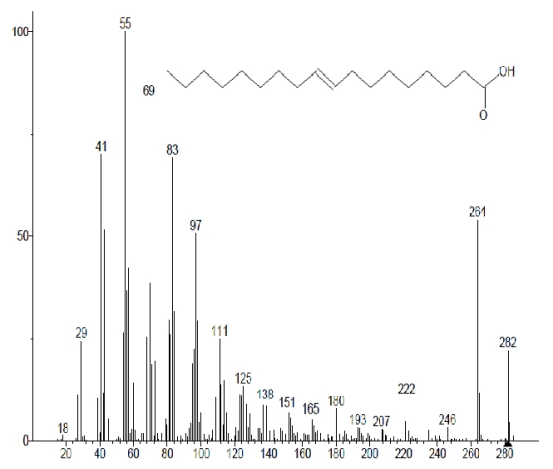

Figure 9: 9-Octadecenoic acid, (E)

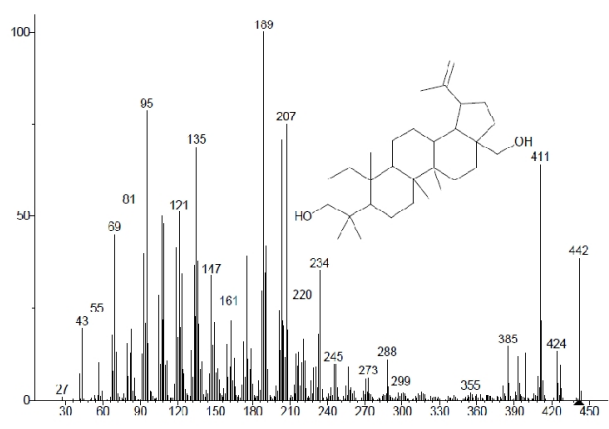

Figure 11: Betulin

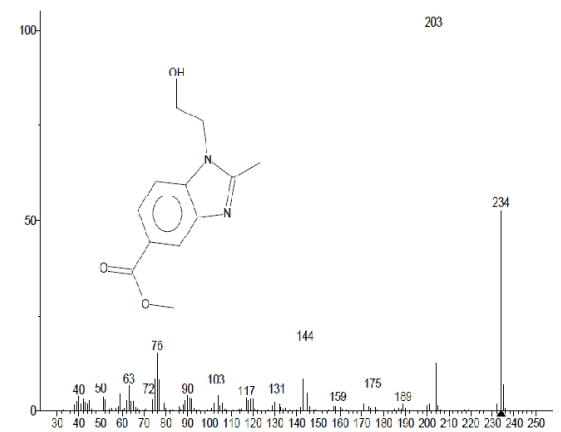

Figure 6: 1-(2-Hydroxy-ethyl)-2-methyl-1H- benzoimidazole-5carboxylic acid methyl ester

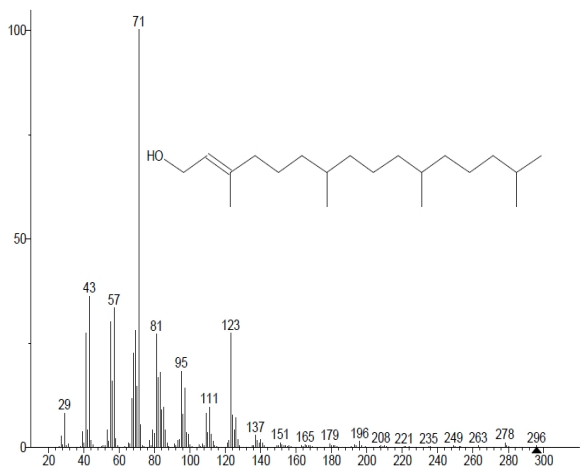

Figure 8: Phytol

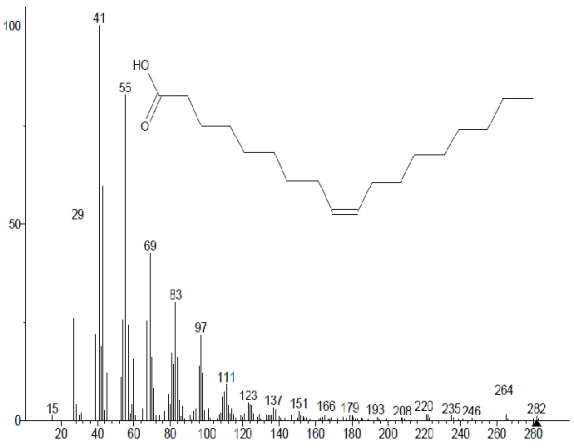

Figure 10: Oleic acid

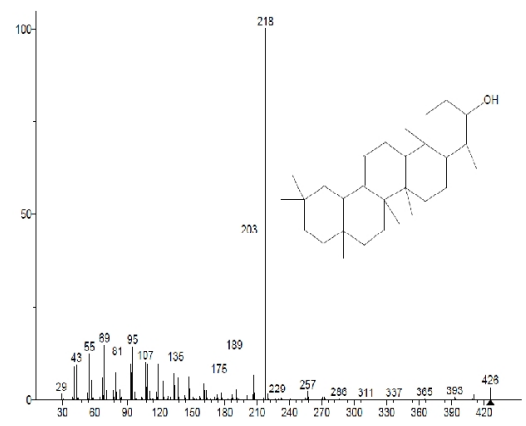

Figure 12: $\beta$-Amyrin 


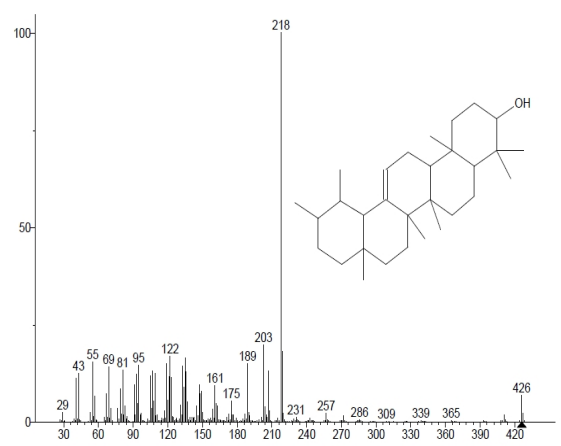

Figure 13: $\alpha$-Amyrin

\section{DISCUSSION}

Among the identified phytochemicals, 9,12-Octadecadienoic acid $(Z, Z)$ have the property of antiinflammatory and antiarthritic as reported by the earlier workers ${ }^{6-8}$. nHexadecanoic have the property of antioxidant and antibacterial activty ${ }^{9}$. n-Hexadecanoic acid has shown cytotoxicity to human leukemic cells, MOLT-4 and also showed in vivo antitumor activity in mice ${ }^{10,11}$. Biological screening tests showed that 2propenoic acid, 3-(4-methoxypheneyl)- have significant activity against different bacteria and fungi ${ }^{12}$. 4-((1E)-3-Hydroxy-1propenyl)-2-methoxyphenol, was used as antimicrobial agents ${ }^{13}$. Pyrazolidine-3, 5-dione derivative has been developed as antiinflammatory and antibacterial agents ${ }^{14}$. Oleic acid may hinder the progression of adreno leuko dystrophy (ALD), a fatal disease that affects the brain and adrenal glands ${ }^{15}$. Oleic acid also keeps cell membranes soft and fluid allowing helpful antiinflammatory substances like omega-3 fatty acid to penetrate the cell membrane move easily and preventing the negative effects of bad cholestrol ${ }^{16}$. It has been reported that 5hydroxymethylfurfural has antioxidant and anti-ischemic properties, and can enhance erythrocyte deformation and alter blood haemorheology in rats ${ }^{17}$. 5-Hydroxymethylfurfural was also found to improve acute liver injury in mice ${ }^{18}$ and could inhibit the oxidative damage to hepatocytes caused by $\mathrm{H}_{2} \mathrm{O}_{2}$ in human $\mathrm{LO}_{2}$ hepatocytes ${ }^{19}$. All these suggest the anti- apoptosis mechanism of 5-hydroxymethylfurfural. A mixture of $\alpha$ - and $\beta$ amyrin isomers protects liver against acetaminophen-induced injuries $^{20}$ and ameliorates 1-arginine-induced acute pancreatitis $^{21}$. $\alpha$-Amyrin esters were also reported to exhibit significant biological activity, namely cytotoxic $^{22}$ and antiinflammatory ${ }^{23}$. Phytol is detected in M. serratulum stem which was also found to be effective in different stages of arthritis. Phytol is a key acyclic diterpene alcohol that is a precursor for vitamins $E$ and $K_{1}$. It was found to give good as well as preventive and therapeutic results against arthritis. The results show that reactive oxygen species promoting substances such as phytol constitute of promising novel class of pharmaceuticals for the treatment of rheumatoid arthritis and possibly chronic inflammatory disease ${ }^{24}$.

\section{CONCLUSION}

The presence of various bioactive compounds justifies the use of this plant for various ailments by traditional practitioners. However, isolation of individual phytochemical constituents and subjecting its biological activity will definitely give fruitful results. It could be concluded that Myxopyrum serratulum stem contains various bioactive compounds. So it is recommended as a plant of phytopharmaceutical importance. However, further

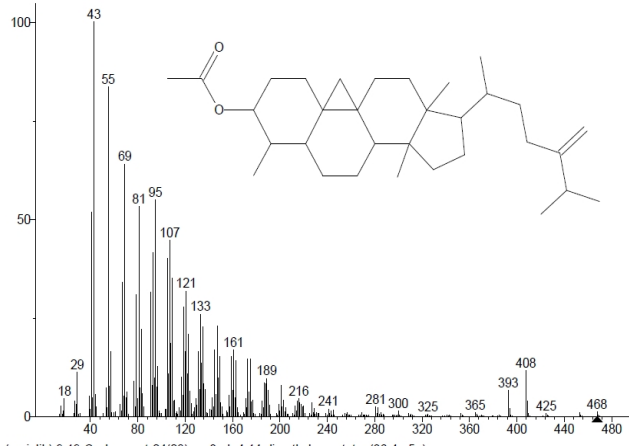

Figure 14: 9,19-Cycloergost-24(28)-en-3-ol, 4,14-dimsethyl-, acetate, $(3 \beta, 4 \alpha, 5 \alpha)-$

studies are needed to undertake its bioactivity and toxicity profile.

\section{ACKNOWLEDGEMENT}

We would like to thank Mr. S. Kumaravel, Senior Scientist, Indian Institute of Crop Processing Technology (Ministry of food Processing Industries, Government of India) Tanjavore, Tamilnadu for providing all the facilities and support used to carry out the work.

\section{REFERENCES}

1. Steinmetz KA, Potter JD. Vegetables, fruits, cancer prevention: A review. Journal of the American Dietetic Association 1996; 96: 1027-1039.

2. Ordache M, Culea C, Gherman O, Cozar O. Characterization of some plant extracts by GC-MS, Nuclear Instruments and Methods in Physical Research Section B: Beam Interactions with Materials and Atoms 2009; 267: 338-342.

3. Priya V, Jananie Rk, Vijayalakshmi K. GC/MS determination of bioactive components of Pleurotus ostreatus. Int. Res. J. Pharm. 2012; 3(3): 150-151.

4. Rajalakshmi K, Mohan VR. Antiinflammatory activity of stem and leaf of Myxopyrum serratulum A.W. Hill (Oleaceae). International Journal of Current Pharmaceutical Review and Research 2016; 7: 190-192.

5. Muthulakshmi A, Margret JR, Mohan VR. GC-MS Analysis of Bioactive components of Feronia elephantum correa (Rutaceae). Journal of Applied Pharmaceutical Science 2012; 2: 69-74.

6. Lalitha rani S, Mohan VR, Rejini GS, Kalidass C. GC-MS analysis of ethanolic extract of Pothos scandens leaf. Journal of Herbal Medicinal Toxicology 2009; 3: 159-160.

7. Maruthupandian A, Mohan VR. GC-MS analysis of ethanol extract of Wattakaka volubilis (L.F). Stapf. leaf. International Journal of Phytomedicine 2011; 3: 59-62.

8. Kala SMJ, Balasubramanian T, Tresina soris P, Mohan VR. GC-MS determination of bioactive compounds of Eugenia singampattiana Bedd. International Journal of ChemTech Research 2011; 3: 1534-1537.

9. Canas Rodriguez A, Smith HW. The identification of the antimicrobial factors of the stomach contents of sucking rabbits. Biochemical Journal 1966; 100: 79-82.

10. Semary NAE, Ghazy SM, Naby MMAE. Investigating the taxonomy and bioactivity of an Egyptian chlorococcum isolate. Australian Journal of Basic Applied Sciences 2009; 3: $1540-1551$.

11. Harada H, Yamashita U, Kurihara H, Fukushi E, Kawabata J, Kamei Y. Antitumor activity of palmitic acid found as a 
selective cytotoxic substance in a marine red alga. Anticancer Research 2002; 22: 2587-2590.

12. Rehman S, Shahid K, Ali S, Mazhar M, Badshah A, Gerorge E, Song X, Ryczkowski J. Synthesis, spectroscopic characterization, and in vitro biological activity of organotin (IV) complexes of (E)-3-(4-methoxyphenyl)-2-phenyl-2propenoic acid. Heteroatom Chemistry 2005; 16: 175-183.

13. Ravikumar VR, Gopal V, sudha T. Analysis of phytochemical constituents of stem bark extracts of Zanthoxylum tetraspermum Wight \& Arn. Research Journal of Pharmaceutical Biological and Chemical Sciences 2012; 3: 391-402.

14. Singh R, Upmanyu N, Gupta M, Prasad P. Novel synthesis, characterization and Biological Evaluation of substituted Pyrazolidine-3,5-Dione. JSM Chemistry 2015; 3: 10161022.

15. "Adrenoleukodystrophy: Oleic acid lowers fibroblast saturated C22-26 fatty acids", National Center for Biotechnology Information, 1986-03-03, Retrieved 2008-1007.

16. Perricone, Nicholas. The Perricone Prescription: A Physician's 28-day Program for Total Body and Face Rejuvenation (1st ed), New York: Harper Resources 2002, 66-67.

17. Wen YZ, Ma L, Huang SF, et al. Determination of 5Hydroxymethylfurfural in Decoction of Rhizoma polygonati. Guangzhou Zhongyiyao Daxue Xuebao 2010; 27(5): 507 509.

18. Ding X, Wang MY, Yu ZL, et al. Studied on separation, appraisal and the biological activity of 5-HMF in Cornus officinalis. Zhongguo Zhong yao Za zhi 2008; 33(4): 392396.

19. Wang MY, Zhao FM, Cai BC, et al. Investigation on the morphological protective effect of 5-hydroxymethylfurfural extracted from wine-processed Fructus corni on human L02 hepatocytes. Journal of Ethnopharmacology 2010; 130(2): 424- 428.

20. Oliveira FA, Chaves MH, Almeida FR, Lima RC Jr, Silva RM, Maia JL, Brito GA,Santos FA, Rao VS. Protective effect of alpha- and beta-amyrin, a triterpene mixture from Protium heptaphyllum (Aubl.) March. trunk wood resin, against acetaminophen-induced liver injury in mice. Journal of Ethnopharmacology 2005; 98: 103-108.

21. Melo CM, Carvalho KM, Neves JC, Morais TC, Rao VS, Santos FA, Brito GA, Chaves MH. Alph-a,beta-amyrin, a natural triterpenoid ameliorates L-arginine-induced acute pancreatitis in rats. World Journal of Gastroenterology 2010; 16: 4272-4280.

22. Barros FW, Bandeira PN, Lima DJ, Meira AS, de Farias SS, Albuquerque MR, Santos HS, Lemos TL, de Morais MO, Costa-Lotufo LV, Pessoa Cdo Ó. Amyrin esters induce cell death by apoptosis in HL-60 leukemia cells. Bioorganic and Medicinal Chemistry 2011; 19: 1268-1276.

23. Akihisa T, Kojima N, Kikuchi T, Yasukawa K, Tokuda H, T Masters E, Manosroi A, Manosroi J. Anti-inflammatory and chemopreventive effects of triterpene cinnamates and acetates from shea fat. Journal of Oleo Science 2010; 59: 273-80.

24. Ogunlesi M, Okiei W, Ofor E, Osibote AE. Analysis of the essential oil from the dried leaves of Euphorbia hirta Linn (Euphorbiaceae), a potential medication for asthma. African Journal of Biotechnology 2009; 8: 7042-7050.

\section{Cite this article as:}

K. Rajalakshmi, V. R. Mohan. Determination of bioactive components of Myxopyrum serratulum A.W. Hill (Oleaceae) stem by GC-MS analysis. Int. Res. J. Pharm. 2016;7(7):36-42 http://dx.doi.org/10.7897/2230-8407.07782

Disclaimer: IRJP is solely owned by Moksha Publishing House - A non-profit publishing house, dedicated to publish quality research, while every effort has been taken to verify the accuracy of the content published in our Journal. IRJP cannot accept any responsibility or liability for the site content and articles published. The views expressed in articles by our contributing authors are not necessarily those of IRJP editor or editorial board members. 